\title{
Aspectos prácticos de la administración de rivaroxabán
}

\author{
Carmen Suárez-Fernández, Vanessa Roldán, José Vivancos
}

\begin{abstract}
Resumen. El rivaroxabán es un inhibidor oral altamente selectivo del factor Xa. Actualmente, el uso de rivaroxabán está aprobado para la prevención del tromboembolismo venoso en adultos a los que se va a realizar un reemplazo electivo tanto de rodilla como de cadera, para el tratamiento de la trombosis venosa profunda y embolia de pulmón y la prevención secundaria a largo plazo del tromboembolismo venoso, y para la prevención del ictus y del embolismo sistémico en pacientes con fibrilación auricular no valvular. El rivaroxabán posee múltiples ventajas sobre los antagonistas de la vitamina K, y esto puede facilitar su uso en la práctica clínica. En consecuencia, es probable que los nuevos anticoagulantes cambien las estrategias de manejo de los pacientes que requieran anticoagulación. Por otra parte, el rivaroxabán tiene algunas particularidades que es necesario conocer. El objetivo de este artículo ha sido revisar el uso del rivaroxabán no solo en la población general, sino también en diferentes subgrupos de pacientes y situaciones clínicas, para así lograr un manejo óptimo de este fármaco en la práctica clínica diaria.
\end{abstract}

Palabras clave. Anticoagulación. Fibrilación auricular. Ictus. Rivaroxabán. Tratamiento. Tromboembolismo venoso. Trombosis venosa profunda.

\section{Introducción}

El rivaroxabán es un nuevo anticoagulante oral, de administración única diaria, que produce una inhibición directa altamente selectiva del factor Xa de la coagulación [1]. Existe un amplio programa de investigación que ha analizado los beneficios que aporta el rivaroxabán en la prevención de las complicaciones tromboembólicas en diferentes escenarios clínicos, entre los que destacan:

- Programa RECORD (Regulation of Coagulation in Orthopedic Surgery to Prevent DVT and PE), compuesto por cuatro ensayos clínicos en fase III, con más de 12.500 pacientes, y que analizó la eficacia y seguridad del rivaroxabán en la prevención de la trombosis venosa profunda (TVP) y el embolismo pulmonar tras cirugía ortopédica [2-5].

- Programa ROCKET-AF (Rivaroxaban Once Daily Oral Direct Factor Xa Inhibition Compared with Vitamin K Antagonism for Prevention of Stroke and Embolism Trial in Atrial Fibrillation), en el que se estudió la eficacia y seguridad del rivaroxabán frente a la warfarina en más de 14.000 pacientes con fibrilación auricular no valvular (FANV) [6].

- Programa EINSTEIN, compuesto por tres ensayos clínicos, el EINSTEIN-DVT, el EINSTEIN-PE y el EINSTEIN-EXTENSION, en los que se ana- lizó la eficacia del rivaroxabán en el tratamiento agudo y la prevención de recurrencias de tromboembolismo venoso en pacientes con TVP sintomática y con embolismo pulmonar agudo $[7,8]$.

Los buenos resultados obtenidos por el rivaroxabán en estos ensayos clínicos han hecho posible que en España se apruebe con las siguientes indicaciones:

- Prevención del tromboembolismo venoso en pacientes adultos sometidos a cirugía electiva de reemplazo de cadera o rodilla.

- Prevención del ictus y de la embolia sistémica en pacientes adultos con FANV, con uno o más factores de riesgo (insuficiencia cardíaca congestiva, hipertensión, edad $\geq 75$ años, diabetes mellitus, ictus o ataque isquémico transitorio previos).

- Tratamiento de la TVP y de la embolia pulmonar y prevención de la TVP recurrente y de la embolia pulmonar en pacientes adultos [1].

Su administración oral única diaria consigue una farmacocinética predecible, que proporciona una anticoagulación constante a lo largo de las 24 horas del día, independientemente de la edad, el sexo, el peso o la raza. Además, presenta escasas interacciones con otros fármacos, y no es necesario realizar controles rutinarios de la coagulación. Asimismo, tiene un comienzo y final de acción rápido, así como una
Servicio de Hematología; Hospital Universitario Morales Meseguer; Murcia (V. Roldán). Servicio de Medicina Interna (C. SuárezFernández); Servicio de Neurología y Unidad de Ictus (J. Vivancos); Instituto de Investigación Sanitaria Princesa; Hospital Universitario La Princesa; Madrid, España.

Correspondencia:

Dra. Carmen Suárez Fernández. Servicio de Medicina Interna. Hospital Universitario La Princesa. Diego de León, 62. E-28006 Madrid.

E-mail:

csuarezf@medynet.com

Declaración de intereses: Los autores no declaran ningún conflicto de interés en relación con este artículo. Content Ed Net ha proporcionado asistencia editorial en la preparación de este manuscrito, la cual ha sido financiada por Bayer.

Aceptado tras revisión externa: 02.04.13.

Cómo citar este artículo: Suárez-Fernández C, Roldán V , Vivancos J. Aspectos prácticos de la administración de rivaroxabán. Rev Neurol 2013; 57: 411-21.

(c) 2013 Revista de Neurología 
Tabla I. Características farmacocinéticas y farmacodinámicas del rivaroxabán (adaptado de [1,9-14]).

\begin{tabular}{|c|c|}
\hline Mecanismo de acción & $\begin{array}{l}\text { Inhibición directa y altamente } \\
\text { electiva del factor Xa }\end{array}$ \\
\hline \multirow{2}{*}{ Biodisponibilidad oral } & $\begin{array}{l}\text { El 80-100\% para rivaroxabán } 10 \text { mg, } \\
\text { independientemente de la ingesta }\end{array}$ \\
\hline & $\begin{array}{l}\text { El } 66 \% \text { para rivaroxabán } 20 \mathrm{mg} \text {. Cuando } \\
\text { se toma con alimentos, aumenta un } 39 \%\end{array}$ \\
\hline Concentración máxima $\left(C_{\text {máx }}\right)$ & 2-4 horas \\
\hline \multirow[t]{2}{*}{ Unión a proteínas plasmáticas } & $92-95 \%$ \\
\hline & $\begin{array}{l}\text { Aproximadamente } 2 / 3 \text { del fármaco } \\
\text { sufren degradación metabólica y } 1 / 3 \text { es } \\
\text { directamente excretado por los riñones }\end{array}$ \\
\hline Metabolismo y eliminación & $\begin{array}{l}\text { El rivaroxabán es metabolizado mediante } \\
\text { el CYP3A4, el CYP2J2 y mecanismos } \\
\text { independientes del CYP. Además, el } \\
\text { rivaroxabán es un sustrato de las proteínas } \\
\text { transportadoras P-gp y Bcrp }\end{array}$ \\
\hline \multirow{2}{*}{ Vida media de eliminación } & 5-9 horas (jóvenes) \\
\hline & 11-13 horas (edad avanzada) \\
\hline
\end{tabular}

\begin{tabular}{|c|c|c|}
\hline \multirow{7}{*}{$\begin{array}{l}\text { Interacciones } \\
\text { relevantes }\end{array}$} & $\begin{array}{l}\text { Antibióticos macrólidos } \\
\text { (claritromicina, eritromicina) }\end{array}$ & $\begin{array}{l}\text { No se han observado interacciones } \\
\text { clínicamente significativas, si bien } \\
\text { hay que tener precaución en caso } \\
\text { de insuficiencia renal }\end{array}$ \\
\hline & $\begin{array}{l}\text { Antiinflamatorios no esteroideos, } \\
\text { antiagregantes, otros agentes } \\
\text { antitrombóticos }\end{array}$ & $\begin{array}{l}\text { Debe tenerse precaución debido } \\
\text { al aumento del riesgo de hemorragia }\end{array}$ \\
\hline & $\begin{array}{l}\text { Midazolam (sustrato de CYP3A4), } \\
\text { digoxina (sustrato de P-gp), atorvastatina } \\
\text { (sustrato de CYP3A4 y P-gp) }\end{array}$ & $\begin{array}{l}\text { No se han observado interacciones } \\
\text { relevantes con rivaroxabán }\end{array}$ \\
\hline & $\begin{array}{l}\text { Inhibidores de la bomba de protones, } \\
\text { antagonistas de los receptores } \mathrm{H} 2 \text {, } \\
\text { antiácidos, zumos de frutas }\end{array}$ & $\begin{array}{l}\text { No se han observado interacciones } \\
\text { relevantes con rivaroxabán }\end{array}$ \\
\hline & $\begin{array}{l}\text { Inductores potentes de CYP3A4 } \\
\text { (carbamacepina, fenitoína, } \\
\text { fenobarbital, hipérico, rifampicina) }\end{array}$ & $\begin{array}{l}\text { Los medicamentos de esta clase deben } \\
\text { administrarse de forma concomitante } \\
\text { con precaución }\end{array}$ \\
\hline & $\begin{array}{l}\text { Inhibidores potentes de CYP3A4 y } \\
\text { P-gp (antimicóticos sistémicos, como } \\
\text { itraconazol, ketoconazol, posaconazol, } \\
\text { voriconazol, o inhibidores de la proteasa } \\
\text { del virus de inmunodeficiencia humana, } \\
\text { como ritonavir) }\end{array}$ & $\begin{array}{l}\text { No se recomienda la coadministración con } \\
\text { rivaroxabán (sin embargo, el fluconazol } \\
\text { se puede administrar con precaución) }\end{array}$ \\
\hline & Dronedarona & $\begin{array}{l}\text { La administración concomitante con } \\
\text { rivaroxabán debería evitarse, debido } \\
\text { a que los datos clínicos son limitados }\end{array}$ \\
\hline
\end{tabular}

ventana terapéutica amplia (Tabla I) [1,9-14]. Aunque el empleo de rivaroxabán se ha estudiado extensamente en ensayos clínicos que avalan su efica- cia y seguridad, al ser un fármaco relativamente nuevo existen aún ciertas dudas en cuanto a su manejo. A continuación se analizarán las más relevantes.

\section{¿Cómo debe administrarse el rivaroxabán?}

La dosificación y duración del tratamiento con rivaroxabán varía según las indicaciones. Para la prevención de tromboembolismo venoso tras cirugía electiva de reemplazo de cadera, se recomienda rivaroxabán $10 \mathrm{mg}$ una vez al día durante cinco semanas, y dos semanas si el reemplazo es de rodilla basándose en los ensayos clínicos. No obstante, la guía de profilaxis tromboembólica de la Sociedad Española de Cirugía Ortopédica y Traumatología recomienda en ambos casos un tratamiento de 4-6 semanas [15]. En ambos casos, el comienzo del tratamiento debe iniciarse entre 6 y 10 horas después de la intervención quirúrgica, siempre que se haya establecido la hemostasia [1,12-14].

Para el tratamiento agudo de la TVP, la dosis recomendada es de $15 \mathrm{mg}$ dos veces al día durante tres semanas y $20 \mathrm{mg}$ una vez al día para la prevención de recurrencias de TVP o embolia pulmonar durante al menos tres meses si la causa del tromboembolismo venoso fuese transitoria (cirugía reciente, trauma...), o mayor duración si ésta fuese idiopática o persistiesen los factores desencadenantes. En los pacientes con insuficiencia renal moderada a grave (aclaramientos de creatinina de $15-49 \mathrm{~mL} / \mathrm{min}$ ), puede considerarse una reducción de esta dosis a $15 \mathrm{mg}$ una vez al día, a partir de las tres semanas iniciales, si el riesgo de hemorragia supera el de recurrencia de tromboembolismo venoso [1,12-14].

En pacientes adultos con FANV y uno o más factores de riesgo, para la prevención de ictus y embolismo sistémico, se recomienda la dosis de $20 \mathrm{mg}$ una vez al día ( $15 \mathrm{mg}$ una vez al día si existe insuficiencia renal con aclaramientos de creatinina de $30-49 \mathrm{~mL} / \mathrm{min}$ ) de manera indefinida y siempre que el riesgo de ictus/embolismo sistémico supere al de sangrado (Tabla II) [1,12-14]. Aunque no se incluyó ningún paciente con aclaramiento de creatinina inferior a $30 \mathrm{~mL} / \mathrm{min}$, se puede administrar $15 \mathrm{mg}$ de rivaroxabán en caso de aclaramiento de creatinina de $15-30 \mathrm{~mL} / \mathrm{min}$ con precaución y siempre que el beneficio supere al riesgo.

Mientras que rivaroxabán $10 \mathrm{mg}$ puede tomarse con o sin alimentos, cuando la dosis es de 15 o $20 \mathrm{mg}$ debe tomarse con alimentos, ya que así aumenta la absorción, y no existen restricciones alimenticias (Tabla I). A diferencia de las frecuentes interacciones farmacológicas con los antagonistas de la vita- 
mina K (AVK), el rivaroxabán presenta escasas interacciones con otros fármacos. Las más relevantes se resumen en la tabla I $[1,9-14,16]$.

\section{¿Qué hacer si el paciente olvida tomar una dosis de rivaroxabán?}

Si el paciente toma rivaroxabán como tratamiento de la TVP y prevención de TVP/embolia pulmonar recurrente después de una TVP aguda, ante el olvido de una dosis durante la fase de tratamiento agudo (días 1-21), en la que debería recibir 15 mg dos veces al día, el paciente debe tomar la dosis olvidada inmediatamente, para garantizar una toma de $30 \mathrm{mg}$ de rivaroxabán al día. En este caso, pueden tomarse dos comprimidos de $15 \mathrm{mg}$ al mismo tiempo, continuando con la toma normal de $15 \mathrm{mg}$ dos veces al día a partir del día siguiente. Si el olvido ocurre durante la fase de prevención secundaria (del día 22 en adelante), el paciente debe tomar rivaroxabán inmediatamente y continuar al día siguiente con la toma una vez al día, según las recomendaciones. No debe duplicarse la dosis el mismo día [1]. Esta misma estrategia se seguirá en el caso de tratamiento por FANV [1]. En caso de vómitos, y dada la absorción rápida de rivaroxabán, si un paciente los presenta más de dos horas después de la toma de rivaroxabán, no debe administrarse ninguna dosis adicional [1].

\section{¿En qué pacientes no puede emplearse el rivaroxabán?}

Existen ciertas circunstancias en las que el uso del rivaroxabán está contraindicado o requieren precaución especial. La hipersensibilidad al principio activo o a alguno de los excipientes, cuando exista una hemorragia activa clínicamente relevante, lesión o enfermedad con un riesgo significativo de sangrado grave (úlcera gastrointestinal activa o reciente, presencia de neoplasias malignas con alto riesgo de sangrado, traumatismo cerebral o espinal reciente, cirugía cerebral, espinal u oftálmica reciente, hemorragia intracraneal reciente, varices esofágicas, malformaciones arteriovenosas, aneurismas o anomalías vasculares intraespinales o intracerebrales clínicamente relevantes, tratamiento concomitante con cualquier otro agente anticoagulante, excepto bajo las circunstancias de cambio de tratamiento a o desde rivaroxabán), contraindica su administración. También está contraindicado en caso de enfermedad hepática asociada a coagulopatía o
Tabla II. Indicaciones actuales de rivaroxabán, dosificación y duración del tratamiento (adaptado de [1,12-14]).

\begin{tabular}{ll}
\hline & $\begin{array}{l}\text { Rivaroxabán } 10 \mathrm{mg} \text { una vez al día } \\
\text { (cinco semanas si es la cadera, dos semanas si es la rodilla) }\end{array}$ \\
\cline { 2 - 2 } $\begin{array}{l}\text { Prevención de TEV } \\
\text { tras cirugía electiva de } \\
\text { reemplazo de cadera/rodilla }\end{array}$ & $\begin{array}{l}\text { En ambos casos, el comienzo del tratamiento debe iniciarse entre } \\
\text { 6-10 horas después de la intervención quirúrgica, siempre que se } \\
\text { haya establecido la hemostasia }\end{array}$ \\
$\begin{array}{ll}\text { Tratamiento agudo de la } \\
\text { TVP proximal y la embolia } \\
\text { pulmonar y prevención } \\
\text { de recurrencias de la TVP } \\
\text { o la embolia pulmonar }\end{array}$ & $\begin{array}{l}\text { Tratamiento agudo: rivaroxabán 15 mg dos veces al día durante tres semanas } \\
\text { tres meses si la causa del TEV fuese transitoria (cirugía reciente, } \\
\text { traumatismo...) o mayor duración si ésta fuese idiopática o persistiesen } \\
\text { los factores desencadenantes. En pacientes con aclaramientos de } \\
\text { creatinina de 15-49 mL/min, puede considerarse una dosis de 15 mg } \\
\text { una vez al día si el riesgo de hemorragia supera el de recurrencia de TEV }\end{array}$ \\
\hline $\begin{array}{l}\text { FANV y } \geq 1 \text { factor de riesgo } \\
\text { Rivaroxabán } 20 \text { mg una vez al día (15 mg una vez al día si hay } \\
\text { aclaramientos de creatinina de 15-49 mL/min) de manera indefinida, } \\
\text { si el riesgo de ictus/embolismo sistémico supera al de sangrado }\end{array}$
\end{tabular}

FANV: fibrilación auricular no valvular; TEV: tromboembolismo venoso; TVP: trombosis venosa profunda. Las evidencias de rivaroxabán en pacientes con un aclaramiento de creatinina de $15-29 \mathrm{~mL} / \mathrm{min}$ son escasas, por lo que en estos pacientes el rivaroxabán debe emplearse con precaución. El rivaroxabán está contraindicado en pacientes con aclaramiento de creatinina $<15 \mathrm{~mL} / \mathrm{min}$.

con riesgo clínicamente significativo de hemorragia (incluyendo a los sujetos con cirrosis Child-Pugh B y C), embarazo o lactancia. Tampoco se recomienda en pacientes con insuficiencia renal muy grave (aclaramiento de creatinina $<15 \mathrm{~mL} / \mathrm{min})$. Del mismo modo, no se recomienda el uso concomitante vía sistémica con inhibidores potentes del CYP3A4 y de las proteínas transportadoras P-gp -antimicóticos azólicos como ketoconazol, itraconazol, voriconazol y posaconazol, e inhibidores de la proteasa del virus de la inmunodeficiencia humana (VIH), como el ritonavir- (Tablas I y III) [1,12-14].

\section{¿Es segura la administración única diaria?}

A diferencia del dabigatrán y el apixabán, que se administran cada 12 horas, el rivaroxabán se administra una vez al día en el tratamiento de la prevención de las complicaciones tromboembólicas en el paciente con FANV $[6,17,18]$. En el estudio ROCKET-AF, se escogió la dosis de $20 \mathrm{mg}$ al día principalmente basándose en dos ensayos clínicos en fase II que investigaban la eficacia y seguridad del rivaroxabán en el tratamiento del tromboembolismo venoso (mecanismo de formación de trombo parecido al de la FANV), en donde se observó que $20 \mathrm{mg}$ una vez al día de rivaroxabán era la dosis que presentaba una mayor eficacia, con un menor riesgo de sangrado. 
Tabla III. Contraindicaciones de rivaroxabán y situaciones en las que se debe emplear con precaución (adaptado de [1]).

En pacientes con hipersensibilidad a rivaroxabán o a alguno de los excipientes

Cuando exista una hemorragia activa clínicamente significativa

Cuando haya lesión o enfermedad con un riesgo significativo de sangrado grave (úlcera gastrointestinal activa o reciente, presencia de neoplasias malignas con alto riesgo de sangrado, traumatismo cerebral o medular reciente, cirugía cerebral, espinal u oftálmica reciente, hemorragia intracraneal reciente, sospecha de varices esofágicas, malformaciones arteriovenosas, aneurismas o

Contraindicaciones anomalías vasculares intraespinales o intracerebrales clínicamente relevantes)

Cuando exista tratamiento concomitante con cualquier otro agente anticoagulante, excepto bajo las circunstancias de cambio de tratamiento a o desde rivaroxabán

En caso de enfermedad hepática asociada a coagulopatía o con riesgo clínicamente significativo de hemorragia (incluyendo a los sujetos con cirrosis Child-Pugh B y C)

En caso de embarazo o lactancia

Uso concomitante de inhibidores potentes del CYP3A4 y de las proteínas transportadoras P-gp (antimicóticos azólicos, como ketoconazol, itraconazol, voriconazol y posaconazol, e inhibidores de la proteasa del virus de inmunodeficiencia humana, como el ritonavir)

Insuficiencia renal avanzada (aclaramiento de creatinina $<15 \mathrm{~mL} / \mathrm{min}$ )

Situaciones en las que debe emplearse con precaución

\section{¿Son importantes las interacciones del rivaroxabán con otros medicamentos?}

Una de las ventajas del rivaroxabán con respecto a los AVK es el escaso número de interacciones que tiene con otros fármacos, lo que facilita su uso en la práctica clínica, particularmente en pacientes polimedicados. Sin embargo, hay ciertos aspectos que es necesario conocer. El rivaroxabán es metabolizado por el CYP3A4 y es un sustrato de la proteína transportadora P-gp (glucoproteína P-I). En consecuencia, aquellos fármacos que inhiban o induzcan de manera significativa a ambos, el CYP3A4 y la $\mathrm{P}$-gp, pueden presentar interacciones relevantes con el rivaroxabán y, por lo tanto, su uso concomitante no se recomienda. Así, por ejemplo, no se recomienda el uso de rivaroxabán en pacientes que reciban tratamiento sistémico concomitante con antimicóticos azólicos (ketoconazol, itraconazol, voriconazol y posaconazol) o con inhibidores de la proteasa del VIH, ya que son inhibidores potentes del CYP3A4 y de la P-gp (Tabla I). Sin embargo, el fluconazol, que es un inhibidor moderado del CYP3A4, a pesar de producir aumento en el área bajo la curva de rivaroxabán, no se considera clínicamente relevante. Los inductores del CYP3A4, como la rifampicina, la fenitoína, la carbamacepina o el fenobarbital, pueden causar una disminución en la concentración plasmática de rivaroxabán, por lo que deben administrarse con precaución. Fuera de estos fármacos, las interacciones del rivaroxabán con otros tratamientos no son clínicamente relevantes (Tabla I) [1,9-14]. farmacocinética han confirmado que el rivaroxabán $20 \mathrm{mg}$, administrado una vez al día, proporciona una actividad anticoagulante adecuada a lo largo de las 24 horas del día [1,6].

Es bien conocido que el cumplimiento terapéutico es fundamental para un adecuado control de las enfermedades crónicas, como la hipertensión arterial, la insuficiencia cardíaca, la cardiopatía isquémica o la FANV, y que uno de los factores que lo facilita es la reducción del número de comprimidos, aspecto que tiene particular importancia en caso de pacientes polimedicados, como pueden ser los candidatos a recibir un tratamiento anticoagulante. La simplificación del tratamiento no solamente mejora la adhesión, sino que se traduce en un mejor pronóstico a largo plazo, así como en una reducción de los costes sanitarios [19-21]. La posibilidad de ser administrado en una única dosis diaria coloca al rivaroxabán en una situación ventajosa en comparación con el dabigatrán y el apixabán en lo que se refiere a facilitar la adhesión y los beneficios que se derivan de ello.

\section{¿Es necesario monitorizar el efecto anticoagulante del rivaroxabán?}

Una de las grandes ventajas del rivaroxabán con respecto a los AVK es que posee un efecto anticoagulante predecible y constante a lo largo de las 24 horas del día, por lo que no es necesario realizar controles de manera periódica, a diferencia de lo que ocurre con los AVK. Esto tiene, sin duda, una repercusión directa en la mejora de la calidad de vida de los pacientes. De hecho, es uno de los aspectos más valorados por los pacientes [1,22-27]. Aunque el tratamiento con rivaroxabán no requiere una monitorización rutinaria de la exposición, ésta puede ser útil en situaciones excepcionales, en las que el conocimiento de la exposición al fármaco puede ayudar en la toma de decisiones clínicas, como, por ejemplo, en caso de sobredosis o cirugía de emergencia [12-14]. A la hora de monitorizar el efecto anticoagulante del rivaroxabán, es impres- 
cindible conocer las características farmacocinéticas del fármaco, principalmente la concentración máxima (2-4 horas después de la ingesta) y la vida media de eliminación (5-13 horas). En consecuencia, es importante conocer el tiempo que hay desde la ingesta del fármaco y el momento en el que se realiza el análisis. En este sentido, el tiempo de protrombina, medido en segundos, podría ser útil, ya que el rivaroxabán modifica el tiempo de protrombina de una manera dependiente de la dosis, con una estrecha correlación con las concentraciones plasmáticas cuando se emplea una tromboplastina de alta sensibilidad, como Neoplastin ${ }^{\circledR}$, para el análisis. La medida de la actividad antifactor Xa al modificarse por el rivaroxabán también podría tener gran interés en este contexto. De hecho, la determinación de la actividad anti-Xa mediante métodos cromogénicos (Technoclone: Technoview; HyphenBiomed: Biophen DiXal; Stago: STA ${ }^{\circledR}$ Rivaroxaban) tendría una mayor sensibilidad y precisión que el tiempo de protrombina. Los resultados se expresan en $\mathrm{ng} / \mathrm{mL}$ de rivaroxabán (Tabla IV) [1,12-14,23-26]. Actualmente, todos estos métodos cuentan con la aprobación de la Unión Europea, se comercializan en Europa y su uso está aprobado en la práctica clínica, aunque no están estandarizados.

\section{¿Cómo debe realizarse el cambio a rivaroxabán en pacientes que están tomando otros anticoagulantes?}

A diferencia de los AVK, el rivaroxabán tiene un inicio de acción rápido. En consecuencia, cuando sea necesario anticoagular a un paciente, se podría comenzar directamente con rivaroxabán sin requerir una terapia puente con heparina. Sin embargo, es importante conocer cómo hacer el cambio de otro anticoagulante a rivaroxabán para asegurar la eficacia y disminuir el riesgo de hemorragias.

Según la ficha técnica del rivaroxabán, en aquellos pacientes que previamente estén tomando AVK, en el caso de FANV, se interrumpirá este tratamiento y se iniciará rivaroxabán cuando la razón normalizada internacional (INR) sea $\leq 3$ (2,5 en el caso de pacientes tratados por TVP y en la prevención de TVP recurrente y embolia pulmonar) [1,12-14] ( Tabla V). Es importante destacar que, al iniciar tratamiento con rivaroxabán, los niveles de INR pueden estar falsamente elevados [1]. De forma específica, también se ha recomendado que si la INR es $<2$, se puede empezar con rivaroxabán de forma inmediata, y si está entre 2 y 2,5 , se debe suspender el fármaco AVK e iniciar rivaroxabán al día siguiente [12].
Tabla IV. Influencia del rivaroxabán en diferentes parámetros analíticos (adaptado de [12])

\begin{tabular}{|c|c|}
\hline \multirow{3}{*}{ Aumento } & Tiempo de tromboplastina parcial activado (TTPa) \\
\hline & Tiempo de protrombina (TP) \\
\hline & Antitrombina anti-Xa \\
\hline \multirow{3}{*}{ Disminución } & TP basado en factores de coagulación (II, V, VII, X) \\
\hline & TTPa basado en factores de coagulación (VIII, IX, XII) \\
\hline & Ensayo de generación de trombina \\
\hline \multirow{2}{*}{ Imprevisible } & Razón normalizada internacional (INR) \\
\hline & Tiempo de coagulación activada \\
\hline \multirow{5}{*}{ Sin efecto } & Fibrinógeno (método Clauss) \\
\hline & Dímero D \\
\hline & Tiempo de trombina \\
\hline & Tiempo de coagulación de ecarina \\
\hline & Antitrombina anti-Ila \\
\hline
\end{tabular}

Si lo que recibe el paciente son heparinas de bajo peso molecular, se iniciará el tratamiento con rivaroxabán 0-2 h antes de la siguiente dosis programada de la heparina, mientras que si recibe heparina no fraccionada intravenosa en perfusión continua, el rivaroxabán se administrará en el momento de suspender la perfusión (Tabla V) [1]. En caso de que el paciente tomara dabigatrán y se requiriera cambiar a rivaroxabán, es necesario tener en cuenta la función renal, dada la predominante eliminación renal del dabigatrán. En la tabla V se muestra cómo proceder según la función renal $[12,13]$.

Igualmente es importante conocer cómo realizar el cambio de rivaroxabán a otros anticoagulantes. En el caso de cambiar de rivaroxabán a AVK, ambos se administrarán simultáneamente hasta que la INR sea al menos de 2, debiéndose realizar la determinación de la INR justo antes de la siguiente dosis de rivaroxabán. Si el cambio requerido es de rivaroxabán a heparina, tanto en el caso de las de bajo peso molecular como en el de las no fraccionadas, éstas se iniciarán en el momento que habría correspondido la siguiente dosis de rivaroxabán. Finalmente, en el caso del dabigatrán, éste se iniciará en el momento que habría correspondido la siguiente dosis de rivaroxabán $[1,12]$. 
Tabla V. Cambio de diferentes tratamientos anticoagulantes a rivaroxabán (adaptado de [1,12-14]).

\begin{tabular}{|c|c|c|}
\hline \multirow{3}{*}{$\begin{array}{l}\text { Antagonistas } \\
\text { de la vitamina } \mathrm{K}\end{array}$} & Ficha técnica [1] y Escolar et al [14] & Beauloye et al [12] \\
\hline & \multirow{2}{*}{$\begin{array}{l}\text { Se interrumpirá el tratamiento con AVK y se } \\
\text { iniciará rivaroxabán cuando la INR sea } \leq 3 \text { en FANV } \\
\text { ( } 2,5 \text { en pacientes tratados por TVP y en la prevención } \\
\text { de TVP recurrente y embolia pulmonar) }\end{array}$} & INR $<2$ : comenzar inmediatamente con rivaroxabán \\
\hline & & INR 2-2,5: se comenzará con rivaroxabán al día siguiente \\
\hline \multirow{3}{*}{ Heparinas } & Ficha técnica [1] & Beauloye et al [12] y Escolar et al [14] \\
\hline & $\begin{array}{l}\text { HBPM (sc): iniciar rivaroxabán 0-2 horas antes de } \\
\text { la siguiente administración programada de la HBPM }\end{array}$ & $\begin{array}{l}\text { HBPM (sc): iniciar rivaroxabán en la siguiente } \\
\text { administración programada de la HBPM }\end{array}$ \\
\hline & $\begin{array}{l}\text { HNF (iv): el rivaroxabán se administrará } \\
\text { en el momento de la suspensión de la HNF }\end{array}$ & $\begin{array}{l}\text { HNF (iv): el rivaroxabán se administrará } \\
\text { inmediatamente después de suspender la HNF }\end{array}$ \\
\hline
\end{tabular}

$\mathrm{CICr} \geq 50 \mathrm{~mL} / \mathrm{min}$ : iniciar rivaroxabán $20 \mathrm{mg}$ una vez al día 12-24 horas después de la última dosis de dabigatrán

Dabigatrán (Beauloye et al [12] y Turpie et al [13])
CICr 30-50 mL/min: iniciar rivaroxabán $15 \mathrm{mg}$ una vez al día 24-48 horas después de la última dosis de dabigatrán

CICr 15-29 mL/min: el dabigatrán está contraindicado. Iniciar rivaroxabán $15 \mathrm{mg}$ una vez al día > 48 horas después de la última dosis de dabigatrán

AVK: antagonistas de la vitamina K; CICr: aclaramiento de creatinina; FANV: fibrilación auricular no valvular; HBPM: heparina de bajo peso molecular; HNF: heparina no fraccionada; INR: razón normalizada internacional; iv: intravenoso; sc: subcutáneo; TVP: trombosis venosa profunda.

\section{¿Qué hacer cuando a un paciente se le va a realizar una intervención quirúrgica?}

Una de las desventajas de los AVK es su lento comienzo y final de acción, lo que obliga a suspender la medicación unos días antes de una intervención quirúrgica y a necesitar una terapia puente, generalmente heparinas de bajo peso molecular, antes y después de aquélla. El rivaroxabán, en cambio, dado su inicio y final de acción rápido, facilita el manejo de la anticoagulación en este contexto. A la hora de valorar cuándo es el mejor momento para suspender el rivaroxabán antes de la intervención quirúrgica, habrá que tener en cuenta tanto el tipo de intervención (riesgo de sangrado) como las características clínicas de cada paciente -riesgo de complicaciones tromboembólicas (en el caso de FANV, puntuación CHADS2, CHA2DS2-VASc), riesgo de sangrado, función renal y hepática, edad, uso concomitante de antiagregantes, entre otros- [1].

Si se trata de intervenciones menores, sin un aumento significativo en el riesgo de sangrado, como una extracción dentaria, podría no ser necesario suspender el rivaroxabán, pero la intervención se realizará al menos tras 18-24 horas de la última dosis de rivaroxabán. La siguiente dosis de rivaroxabán se retrasará hasta que la hemostasia se haya asegurado (Tabla VI) [12].
En general, para la realización de un procedimiento invasivo o una intervención quirúrgica programada, teniendo en cuenta que la vida media de eliminación del rivaroxabán es de 5-9 horas en personas jóvenes, y de 11-13 horas en personas de edad avanzada, se deberá suspender el rivaroxabán, siempre que sea posible, al menos 24 horas antes de la intervención. En aquellas cirugías con un riesgo elevado de sangrado, y valorando la situación clínica del paciente, algunos autores aconsejan suspender 48 horas antes del procedimiento, aunque en la ficha técnica del producto únicamente se contemplan las 24 horas (Tabla VI) $[1,12,26,28]$. En cuanto a su reinicio, éste se realizará lo antes posible, siempre que se haya alcanzado la hemostasia y el riesgo de sangrado sea bajo. Dependiendo de los casos, algunos autores señalan que un rango de 6-24 horas tras la intervención parece adecuado [12,26,28]. En caso de que la cirugía haya sido de alto riesgo hemorrágico, puede ser necesario no iniciar el tratamiento con rivaroxabán pasadas 48 horas; en este caso se puede valorar utilizar una heparina de bajo peso molecular en dosis profilácticas.

En caso de que la intervención sea urgente, se valorará el riesgo de hemorragia frente al de la urgencia de la intervención. Si es posible, la cirugía debería posponerse al menos 24 horas tras la última ingesta de rivaroxabán. Si no, al menos 12 horas 
Tabla VI. Manejo de rivaroxabán ante una intervención quirúrgica o procedimiento invasivo (adaptado de [1,12]).

\begin{tabular}{|c|c|c|c|c|}
\hline & \multicolumn{3}{|c|}{ Cirugía electiva } & \multirow[b]{2}{*}{ Cirugía urgente } \\
\hline & $\begin{array}{l}\text { Intervenciones menores } \\
\text { (sin riesgo de hemorragia) }\end{array}$ & $\begin{array}{l}\text { Intervenciones mayores } \\
\text { (riesgo de sangrado estándar) }\end{array}$ & $\begin{array}{l}\text { Intervenciones mayores } \\
\text { (riesgo elevado de sangrado) }\end{array}$ & \\
\hline $\begin{array}{l}\text { Suspensión de rivaroxabán } \\
\text { antes de la cirugía }\end{array}$ & $\begin{array}{l}\qquad 18 \text { horas } \\
\text { (alternativamente, } \geq 24 \text { horas) }\end{array}$ & $\geq 24$ horas & $\geq 24$ horas $0 \geq 48$ horas $^{a}$ & 24 horas preferiblemente \\
\hline $\begin{array}{l}\text { Reinicio de rivaroxabán } \\
\text { después de la cirugía }\end{array}$ & $\begin{array}{c}\text { Hemostasia conseguida } \\
\text { (alternativamente, } \geq 24 \text { horas) }\end{array}$ & $\begin{array}{c}\text { Hemostasia conseguida } \\
\text { (alternativamente, } \geq 24 \text { horas) }\end{array}$ & $\begin{array}{l}\text { Hemostasia conseguida } \\
\text { (alternativamente, } \geq 24-48 \text { horas) a }\end{array}$ & $\begin{array}{c}\text { Hemostasia conseguida } \\
\text { (alternativamente, } \geq 24-48 \text { horas) a }\end{array}$ \\
\hline
\end{tabular}

desde la última dosis. Si esto no es posible, se podrá evaluar la actividad anticoagulante mediante el tiempo de protrombina utilizando una tromboplastina de alta sensibilidad (Neoplastin ${ }^{\circledR}$ ) o medir la actividad antifactor Xa: si la actividad anticoagulante es baja, se podrá realizar la intervención; en cambio, si ésta es alta y la intervención no puede retrasarse, se podría tratar al paciente con complejo protrombínico (Tabla VI) $[1,12,26,28]$. En un estudio realizado en voluntarios sanos, la administración de complejo protrombínico fue capaz de revertir de manera completa e inmediata el efecto anticoagulante del rivaroxabán [29].

Cuando se aplica anestesia neuraxial (anestesia epidural o espinal) o se realiza una punción lumbar o epidural, los pacientes tratados con rivaroxabán tienen riesgo aumentado de presentar un hematoma epidural o espinal. Por este motivo, el catéter epidural en pacientes que se someten a reemplazo de cadera o rodilla no deberá retirarse antes de 18 horas después de la última administración de rivaroxabán. La siguiente dosis de rivaroxabán se administrará en un plazo no inferior a seis horas después de la retirada del catéter [1].

\section{Pacientes con FANV y síndrome coronario agudo}

La presencia de cardiopatía isquémica es frecuente en el paciente con FANV. De hecho, está presente hasta en el 20\% de los pacientes con FANV [30]. En el estudio ROCKET-AF, la incidencia de infarto de miocardio fue similar en ambos grupos de tratamiento: 0,9 y $1,1 \%$ por año, con rivaroxabán y warfarina, respectivamente (hazard ratio $=0,81$; intervalo de confianza al 95\%, $=0,63-1,06 ; p=0,12$ ) [6].

Los pacientes con FANV que presenten un síndrome coronario agudo mientras están tomando ri- varoxabán deberán ser tratados de acuerdo con la práctica clínica habitual. Debe suspenderse el tratamiento con rivaroxabán en el marco del síndrome coronario agudo y realizarse los procedimientos habituales, como intervencionismo coronario percutáneo, cirugía de revascularización coronaria o trombólisis, según sea necesario. Tras un síndrome coronario agudo o intervencionismo coronario percutáneo en pacientes con FANV con riesgo moderado a alto de ictus, el empleo de la triple terapia, anticoagulación oral (es decir, AVK con una INR reducida de 2-2,5), ácido acetilsalicílico y un inhibidor-P2Y12 (ticlopidina, clopidogrel, prasugrel o ticagrelor) es, a menudo, inevitable. Una vez que la triple terapia se suspenda, se podrá volver a administrar rivaroxabán $20 \mathrm{mg}$ una vez al día (15 mg una vez al día para pacientes con insuficiencia renal moderada o grave) con ácido acetilsalicílico (o clopidogrel). En la actualidad, no existe experiencia en la combinación de una dosis completa de rivaroxabán (20 mg una vez al día) con la terapia antiplaquetaria dual [13].

\section{Rivaroxabán en pacientes con insuficiencia renal}

La insuficiencia renal es frecuente en pacientes con FANV. Así, algo más del 10\% de pacientes con FANV atendidos en atención primaria presenta, además, insuficiencia renal [30]. La insuficiencia renal aumenta tanto el riesgo de ictus como de sangrado en pacientes con FANV [31]. Por lo tanto, cuando es necesario anticoagular a pacientes con insuficiencia renal, es fundamental emplear aquellos anticoagulantes que han demostrado ser más seguros en este contexto. En el estudio ROCKET-AF, los pacientes con un aclaramiento de creatinina de $30-49 \mathrm{~mL} / \mathrm{min}$ (20,7\% del total) recibieron $15 \mathrm{mg}$ de rivaroxabán 
una vez al día, en lugar de $20 \mathrm{mg}$, o warfarina. A pesar de ser una población de mayor riesgo (se registraron mayor número de episodios vasculares en ambos brazos de tratamiento), la incidencia de ictus o embolismo sistémico fue similar en ambos grupos, si bien hemorragias mortales fueron menos frecuentes en el grupo de rivaroxabán $(0,28 \%$ frente a $0,74 \%$ por 100 pacientes-año; $p=0,047$ ) [32].

Según la ficha técnica, en pacientes con insuficiencia renal leve (aclaramiento de creatinina de $50-80 \mathrm{~mL} / \mathrm{min}$ ) no se requiere ajuste de dosis. En cambio, en pacientes con insuficiencia renal moderada (aclaramiento de creatinina de $30-49 \mathrm{~mL} / \mathrm{min}$ ) o grave (aclaramiento de creatinina de $15-29 \mathrm{~mL} / \mathrm{min}$ ), la dosis recomendada para la prevención de ictus y embolia sistémica en pacientes con FANV es de 15 mg una vez al día; $y$, para el tratamiento de la TVP y la prevención de TVP recurrente y embolia pulmonar, la dosis recomendada es de $15 \mathrm{mg}$ dos veces al día durante las tres primeras semanas. Después se recomienda $20 \mathrm{mg}$ una vez al día, siempre y cuando el riesgo de hemorragia no supere al de recurrencia de tromboembolismo venoso; en el supuesto de que el riesgo de hemorragia superase al de recurrencia de tromboembolismo venoso, puede considerarse una reducción de esta dosis a $15 \mathrm{mg}$ una vez al día. No obstante, la información en los pacientes con un aclaramiento de creatinina de $15-29 \mathrm{~mL} / \mathrm{min}$ es limitada, por lo que obliga a extremar la prudencia y a realizar un seguimiento muy próximo en caso de utilizarse. Finalmente, no se recomienda el empleo del rivaroxabán en sujetos con un aclaramiento de creatinina $<15 \mathrm{~mL} / \mathrm{min}[1]$.

\section{Rivaroxabán en pacientes con insuficiencia hepática}

Los diferentes ensayos clínicos realizados con rivaroxabán no han mostrado un aumento de transaminasas en comparación con enoxaparina o warfarina. En los pacientes cirróticos con insuficiencia hepática leve (Child-Pugh A), se producen cambios menores en la farmacocinética del rivaroxabán, mientras que en aquellos con insuficiencia hepática moderada (Child-Pugh B), el área bajo la curva media de rivaroxabán aumenta significativamente en 2,3 veces, en comparación con voluntarios sanos. En consecuencia, el rivaroxabán puede usarse en pacientes con insuficiencia hepática leve (ChildPugh A), salvo si hay coagulopatía, mientras que no se recomienda su uso en pacientes con daño hepático asociado a coagulopatía o un riesgo relevante de sangrado (Child-Pugh B y C) [1,12-14].

\section{¿Es seguro el rivaroxabán en ancianos?}

Los pacientes de edad avanzada presentan un área bajo la curva que es 1,5 veces superior a los sujetos más jóvenes. Asimismo, mientras que la vida media de eliminación del rivaroxabán es de 5-9 horas en personas jóvenes, ésta asciende a 11-13 horas en los sujetos de edad avanzada. Sin embargo, no es necesario un ajuste de la dosis en los ancianos, salvo que exista un deterioro significativo de la función renal, actuando entonces según las pautas anteriormente descritas [1]. En el ROCKET-AF, la edad media de los pacientes fue de 73 años, y más del $40 \%$ de los pacientes incluidos al menos tenían 75 años. Los resultados de eficacia y seguridad del estudio en los sujetos $\geq 75$ años fueron consistentes con los observados en el resto de pacientes incluidos [6].

\section{¿Qué hacer cuando un paciente sangra estando en tratamiento con rivaroxabán?}

Aunque en el estudio ROCKET-AF se demostró que el rivaroxabán se asociaba con un menor riesgo de hemorragias intracraneales y hemorragias mortales en comparación con la warfarina, el rivaroxabán no deja de ser un anticoagulante, por lo que el riesgo de sangrado es mayor que en los pacientes no anticoagulados. La mayoría de los sangrados referidos con rivaroxabán son leves y, sobre todo, digestivos y genitourinarios. Es importante conocer cuál debe ser su manejo en caso de producirse [6]. Asimismo, también es importante recordar que la concentración máxima de rivaroxabán se alcanza a las 2-4 horas de la ingesta del fármaco, que la vida media de eliminación es de 5-13 horas, y que no existe un antídoto específico y tampoco es dializable [1]. Como norma general, ante un paciente con hemorragia en tratamiento con rivaroxabán, habrá que valorar el estado hemodinámico del paciente, realizar una analítica sanguínea, que incluya hemograma, función renal y pruebas de coagulación, que en el caso del rivaroxabán deberán incluir el tiempo de protrombina o la determinación de la actividad anti-Xa, así como conocer a qué hora se produjo la última ingesta del fármaco [33].

Cuando la hemorragia es leve, sería suficiente retrasar la siguiente dosis o interrumpir el tratamiento temporalmente, junto con medidas de soporte o sintomáticas (compresión mecánica...). Si la hemorragia es moderada o grave, además de interrumpir temporalmente el tratamiento con rivaroxabán y de administrar carbón activado, si la ingesta de rivaroxabán ha sido reciente, se realizarán medidas de 
soporte o sintomáticas (compresión mecánica, endoscopia, ligadura quirúrgica...), así como medidas encaminadas a la reposición de volumen y transfusión de hematíes o de plasma fresco congelado, en caso de anemia o coagulopatía. $\mathrm{Si}$, a pesar de estas medidas, la hemorragia no se controla o supone un riesgo vital, se podría emplear concentrado de complejo de protrombina $[1,12,14,33]$.

En caso de sobredosis, pero sin sangrado, lo primero que hay que recordar es que la absorción en dosis supraterapéuticas es limitada. Si el paciente se encuentra dentro de las 2-4 horas de la ingesta de rivaroxabán, el carbón activado podría ser útil. También puede ayudar en el manejo de estos pacientes la determinación del tiempo de protrombina o de la actividad anti-Xa [12].

\section{¿Cómo actuar si un paciente presenta un infarto cerebral estando en tratamiento con rivaroxabán?}

A pesar de que el rivaroxabán ha demostrado ser eficaz y seguro en pacientes con FANV, un pequeño porcentaje de pacientes tratados con rivaroxabán desarrollará un infarto cerebral. Por otra parte, los resultados del estudio ROCKET-AF fueron similares independientemente de que el paciente hubiera tenido otro ictus o ataque isquémico transitorio previo (prevención secundaria), lo que indica que el rivaroxabán es útil en la prevención tanto de un primer episodio de ictus como de las recurrencias de ictus en pacientes con FANV [34].

Cuando un paciente en tratamiento con rivaroxabán sufre un ictus, se interrumpirá el tratamiento y se realizará de forma urgente una prueba de neuroimagen (tomografía axial computarizada o resonancia magnética craneal) para saber si se trata de un ictus isquémico o hemorrágico. Si el ictus es isquémico, se realizarán los cuidados habituales en este tipo de situaciones. En cuanto a la posibilidad del empleo de tratamiento trombolítico intravenoso con activador del plasminógeno tisular recombinante, si hubiesen transcurrido más de 24 horas desde la última toma de rivaroxabán, se podría considerar este tratamiento en caso de que se cumplieran los criterios requeridos. Sin embargo, si el tiempo transcurrido desde la última toma de rivaroxabán fuera inferior a 24 horas, la seguridad de la trombólisis intravenosa no se ha establecido y habría que considerar como primera opción otras posibilidades terapéuticas, como el tratamiento endovascular mediante trombectomía mecánica $[12,35,36]$.
Es importante conocer la posible causa del ictus (mala adhesión al tratamiento anticoagulante, enfermedad carotídea, ictus lacunar, etc.), para poder establecer el tratamiento específico adecuado. Dado el perfil de eficacia y seguridad de rivaroxabán, este fármaco es una alternativa adecuada para la prevención secundaria del ictus cardioembólico asociado a la FANV. Ahora bien, ¿cuál es el mejor momento para restaurar el tratamiento con rivaroxabán en un paciente que ha tenido un ictus o un ataque isquémico transitorio? En primer lugar, el rivaroxabán no deberá administrarse mientras persista el riesgo de transformación hemorrágica del ictus. Por otra parte, hay que recordar que en el estudio ROCKET-AF fueron criterios de exclusión la presencia de un ictus grave o incapacitante (puntuación Rankin 4-5) en los tres meses previos a la aleatorización, o cualquier ictus en los 14 días antes de la aleatorización, $\mathrm{o}$ ataque isquémico transitorio en los tres días previos a la aleatorización [6]. Aunque siempre habrá que individualizar la necesidad del inicio de la anticoagulación en el caso de un ataque isquémico transitorio que no asociase infarto cerebral establecido, el rivaroxabán podría administrarse tan pronto como fuese posible, mientras que en el caso de un infarto cerebral establecido, de gravedad leve a moderada, pequeño volumen y sin transformación hemorrágica, se podría plantear el inicio de rivaroxabán a partir de los siete días de evolución y esperar al menos tres semanas en casos más graves, mayor volumen o con transformación hemorrágica. En este último caso es aconsejable realizar una prueba de imagen de control para monitorizar la evolución del sangrado [12,35,36].

Aunque el tratamiento con rivaroxabán se asocia con una reducción del 33\% en el riesgo de hemorragia intracraneal con respecto a la warfarina, evidentemente, ésta puede producirse. Aunque la vida media del rivaroxabán es de 5-13 horas, ante un paciente con hemorragia intracraneal es imprescindible revertir su efecto anticoagulante. Para ello, se suspenderá el tratamiento con rivaroxabán y se administrará un concentrado de complejo protrombínico. La decisión de reintroducir el tratamiento anticoagulante es compleja y dependerá de numerosos factores, como el riesgo tromboembólico del paciente, el riesgo de recurrencia de la hemorragia intracraneal, así como de las secuelas que tenga el paciente, por lo que debe ser individualizada. En cualquier caso, no está establecida la seguridad del rivaroxabán en pacientes que ya han sufrido una hemorragia intracraneal, ni tampoco el período óptimo que ha de transcurrir tras la hemorragia para reintroducir el fármaco [12,35-37]. 


\section{Cardioversión en un paciente tratado con rivaroxabán}

Dado que en el estudio ROCKET-AF la cardioversión planificada o ablación fueron criterios de exclusión, la experiencia clínica en este tipo de pacientes es limitada, por lo que no existen recomendaciones clínicas claras en este contexto $[1,12]$. Al igual que con los AVK y las heparinas de bajo peso molecular, la duración y el nivel de anticoagulación deberían evaluarse antes de considerar una cardioversión. En un paciente cumplidor, tras varias semanas de tratamiento, podría efectuarse la cardioversión sin ser necesario detener el tratamiento, teniendo en cuenta que el rivaroxabán es un agente de acción corta. La anticoagulación debería mantenerse durante al menos cuatro semanas después de la cardioversión, independientemente del riesgo basal de ictus. El cambio a un tratamiento apropiado con AVK podría ser una alternativa, hasta disponer de más datos prospectivos de seguridad y eficacia [12].

\section{Conclusiones}

El rivaroxabán en un inhibidor altamente selectivo del factor Xa de la coagulación, que produce una anticoagulación mantenida y predecible a lo largo de las 24 horas del día. Diferentes ensayos clínicos han demostrado la eficacia y seguridad del rivaroxabán en diferentes contextos clínicos, como son la prevención del tromboembolismo venoso en pacientes adultos sometidos a cirugía electiva de reemplazo de cadera o rodilla, la prevención de ictus y embolia sistémica en sujetos con FANV, así como en el tratamiento de la TVP y prevención de la TVP recurrente y de la embolia pulmonar después de una TVP. Además, el rivaroxabán tiene otras ventajas, entre las que destacan su seguridad en pacientes con deterioro de función renal, el escaso número de interacciones con otros fármacos y, en comparación con los otros nuevos anticoagulantes orales, su administración diaria en una sola toma, lo que puede facilitar el cumplimiento terapéutico y la calidad de vida del paciente.

Sin embargo, a pesar de poseer numerosas ventajas frente al tratamiento anticoagulante estándar, al ser un fármaco de reciente inclusión en la práctica clínica, pueden existir ciertas dudas en cuanto a su manejo en determinadas circunstancias conflictivas. El motivo de esta revisión ha sido, precisamente, tratar de clarificar al máximo estas situaciones.

Por otra parte, es importante destacar que existen una serie de incertidumbres con el uso de los nuevos anticoagulantes, como puede ser la adhesión terapéutica; en general, la adhesión al tratamiento con AVK es elevada, al precisar controles periódicos; sin embargo, en el caso de los nuevos anticoagulantes, desconocemos si, al no precisar controles periódicos, el paciente puede discontinuar el tratamiento y el médico no saberlo. En consecuencia, para asegurar una anticoagulación adecuada es imprescindible la educación e información al paciente, quien debe conocer la importancia que tiene tanto la enfermedad que padece como la que se pretende prevenir, así como la necesidad de un buen cumplimiento por su parte. Teniendo en cuenta estas consideraciones, parece claro que los nuevos anticoagulantes orales y, en concreto, el rivaroxabán, ayudarán a simplificar el manejo de la anticoagulación en estos pacientes.

\section{Bibliografía}

1. Agencia Europea del Medicamento (EMEA). Ficha técnica de Xarelto ${ }^{\circledR}$. URL: http://www.ema.europa.eu/docs/es_ES/ document_library/EPAR__Product_Information/human/ 000944/WC500057108.pdf. [20.01.2012].

2. Eriksson BI, Borris LC, Friedman RJ, Haas S, Huisman MV, Kakkar AK, et al; RECORD1 Study Group. Rivaroxaban versus enoxaparin for thromboprophylaxis after hip arthroplasty. N Engl J Med 2008; 358: 2765-5.

3. Kakkar AK, Brenner B, Dahl OE, Eriksson BI, Mouret P, Muntz J, et al; RECORD2 investigators. Extended duration rivaroxaban versus short-term enoxaparin for the prevention of venous thromboembolism after total hip arthroplasty: a double-blind, randomised controlled trial. Lancet 2008; 372: 31-9.

4. Lassen MR, Ageno W, Borris LC, Lieberman JR, Rosencher N, Bandel TJ, et al; RECORD3 Investigators. Rivaroxaban versus enoxaparin for thromboprophylaxis after total knee arthroplasty. N Engl J Med 2008; 358: 2776-86.

5. Turpie AG, Lassen MR, Davidson BL, Bauer KA, Gent M, Kwong LM, et al; RECORD4 Investigators. Rivaroxaban versus enoxaparin for thromboprophylaxis after total knee arthroplasty (RECORD4): a randomised trial. Lancet 2009; 373: 1673-80.

6. Patel MR, Mahaffey KW, Garg J, Pan G, Singer DE, Hacke W, et al; ROCKET AF investigators. Rivaroxaban versus warfarin in nonvalvular atrial fibrillation. N Engl J Med 2011; 365: 883-91.

7. EINSTEIN investigators, Bauersachs R, Berkowitz SD, Brenner B, Buller HR, Decousus H, Gallus AS, et al. Oral rivaroxaban for symptomatic venous thromboembolism. N Engl J Med 2010; 363: 2499-510.

8. EINSTEIN-PE investigators, Büller HR, Prins MH, Lensin AW, Decousus H, Jacobson BF, Minar E, et al. Oral rivaroxaban for the treatment of symptomatic pulmonary embolism. N Engl J Med 2012; 366: 1287-97.

9. Kreutz R. Pharmacodynamic and pharmacokinetic basics of rivaroxaban. Fundam Clin Pharmacol 2012; 26: 27-32.

10. Xu XS, Moore K, Burton P, Stuyckens K, Mueck W, Rossenu S, et al. Population pharmacokinetics and pharmacodynamics of rivaroxaban in patients with acute coronary syndromes. Br J Clin Pharmacol 2012; 74: 86-97.

11. Mueck W, Lensing AW, Agnelli G, Decousus H, Prandoni P, Misselwitz F. Rivaroxaban: population pharmacokinetic analyses in patients treated for acute deep-vein thrombosis and exposure simulations in patients with atrial fibrillation treated for stroke prevention. Clin Pharmacokinet 2011; 50: 675-86. 12. Beauloye C, Dogné JM, Douxfils J, Goethals M, Hainaut P, 
Heidbuchel H, et al. Rivaroxaban. A practical guide V1.0. URL: http://www.thrombosisguidelinesgroup.be/sites/ default/files/Rivaroxaban\%20Practical\%20Guide\%20V1\%20 0_06-07-2012.pdf. [06.07.2012].

13. Turpie AG, Kreutz R, Llau J, Norrving B, Haas S. Management consensus guidance for the use of rivaroxaban -an oral, direct factor Xa inhibitor. Thromb Haemost 2012; 108: 876-86.

14. Escolar-Albadalejo G, García-Frade J, López-Fernández MF Roldán-Schilling V. Guía sobre los nuevos anticoagulantes orales (Sociedad Española de Hematología y Hemoterapia/ Sociedad Española de Trombosis y Hemostasia). URL: http://www.sehh.es/documentos_utilidad_profesional.php.

15. Peidro L, Granero J. Addendum II. In Castellet E, Gomar F, Granero J, Otero R, Peidro L, eds. Guía de profilaxis tromboembólica en cirugía ortopédica y traumatológica. Grupo de estudio de tromboembolismo de la SECOT. Madrid: Sociedad Española de Cirugía Ortopédica y Traumatología; 2009. p. 7-18.

16. Kubitza D, Becka M, Zuehlsdorf M, Mueck W. Effect of food, an antacid, and the $\mathrm{H} 2$ antagonist ranitidine on the absorption of BAY 59-7939 (rivaroxaban), an oral, direct factor Xa inhibitor, in healthy subjects. J Clin Pharmacol 2006; 46: 549-58.

17. Connolly SJ, Ezekowitz MD, Yusuf S, Eikelboom J, Oldgren J, Parekh A, et al. Dabigatran versus warfarin in patients with atrial fibrillation. N Engl J Med 2009; 361: 1139-51.

18. Granger CB, Alexander JH, McMurray JJ, Lopes RD, Hylek EM, Hanna M, et al. Apixaban versus warfarin in patients with atrial fibrillation. N Engl J Med 2011; 365: 981-92.

19. Bangalore S, Kamalakkannan G, Parkar S, Messerli FH. Fixed-dose combinations improve medication compliance: a meta-analysis. Am J Med 2007; 120: 713-9.

20. Dezii CM. A retrospective study of persistence with single-pill combination therapy vs. concurrent two-pill therapy in patients with hypertension. Manag Care 2000; 9 (Suppl 9): S2-6.

21. Frishman WH. Importance of medication adherence in cardiovascular disease and the value of once-daily treatment regimens. Cardiol Rev 2007; 15: 257-63.

22. Schulman S, Crowther MA. How I treat with anticoagulants in 2012: new and old anticoagulants, and when and how to switch. Blood 2012; 119: 3016-23.

23. Madlener K, Hamm C. New anticoagulant drugs for atrial fibrillation. Feasibility and necessity of monitoring. Herz 2012; 37: 378-83.

24. Pollack CV Jr. New oral anticoagulants in the ED setting: a review. Am J Emerg Med 2012; 30: 2046-54

25. Samama MM, Contant G, Spiro TE. Evaluation of the anti-factor Xa chromogenic assay for the measurement of rivaroxaban plasma concentrations using calibrators and controls. Thromb Haemost 2012; 107: 379-87.

26. Levy JH, Key NS, Azran MS. New oral anticoagulants: implications in the perioperative setting. Anesthesiology 2010; 113: 726-45.

27. González-Rojas N, Giménez E, Fernández MA, Heineger AI,
Martínez JL, Villar J, et al. Preferencias por los tratamientos anticoagulantes orales para la prevención a medio y largo plazo del ictus en la fibrilación auricular no valvular. Rev Neurol 2012; 55: 11-9.

28. Sie P, Samama CM, Godier A, Rosencher N, Steib A, Llau JV, et al. Surgery and invasive procedures in patients on long term treatment with direct oral anticoagulants: thrombin or factor Xa inhibitors. Recommendations of the Working Group on perioperative haemostasis and the French Study Group on thrombosis and haemostasis. Arch Cardiovasc Dis 2011; 104: 669-76.

29. Eerenberg ES, Kamphuisen PW, Sijpkens MK, Meijers JC, Buller HR, Levi M. Reversal of rivaroxaban and dabigatran by prothrombin complex concentrate: a randomized, placebocontrolled, crossover study in healthy subjects. Circulation 2011; 124: 1573-9.

30. Barrios V, Calderón A, Escobar C, De la Figuera M. Pacientes con fibrilación auricular asistidos en consultas de atención primaria. Estudio Val-FAAP. Rev Esp Cardiol 2012; 65: 47-53.

31. Hart RG, Eikelboom JW, Ingram AJ, Herzog CA. Anticoagulants in atrial fibrillation patients with chronic kidney disease. Nat Rev Nephrol 2012; 8: 569-78.

32. Fox KA, Piccini JP, Wojdyla D, Becker RC, Halperin JL, Nessel CC, et al. Prevention of stroke and systemic embolism with rivaroxaban compared with warfarin in patients with non-valvular atrial fibrillation and moderate renal impairment. Eur Heart J 2011; 32: 2387-94.

33. Camm AJ, Lip GY, De Caterina R, Savelieva I, Atar D, Hohnloser SH, et al. 2012 focused update of the ESC guidelines for the management of atrial fibrillation: an update of the 2010 ESC guidelines for the management of atrial fibrillation. Eur Heart J 2012; 33: 2719-47.

34. Hankey GJ, Patel MR, Stevens SR, Becker RC, Breithardt G, Carolei A, et al; ROCKET AF Steering Committee investigators. Rivaroxaban compared with warfarin in patients with atrial fibrillation and previous stroke or transient ischaemic attack: a subgroup analysis of ROCKET AF. Lancet Neurol 2012; 11: 315-22.

35. Furie KL, Kasner SE, Adams RJ, Albers GW, Bush RL, Fagan SC, et al. American Heart Association Stroke Council, Council on Cardiovascular Nursing, Council on Clinical Cardiology, and Interdisciplinary Council on Quality of Care and Outcomes Research. Guidelines for the prevention of stroke in patients with stroke or transient ischemic attack: a guideline for healthcare professionals from the American Heart Association/American Stroke Association. Stroke 2011; 42: 227-76.

36. Alonso de Leciñana M, Egido JA, Casado I, Ribó M, Dávalos A, Masjuan J, et al.; por el Comité ad hoc del GEECV de la SEN. Guía para el manejo del infarto cerebral agudo. Neurologia 2011; Dec 6. [Epub ahead of print].

37. Kazmi RS, Lwaleed BA. New anticoagulants: how to deal with treatment failure and bleeding complications. Br J Clin Pharmacol 2011; 72: 593-603.

\section{Practical issues with the use of rivaroxaban}

Summary. Rivaroxaban is an oral highly selective direct factor Xa inhibitor. Rivaroxaban is currently approved for the prevention of venous thromboembolism in adult patients undergoing elective hip or knee replacement surgery, for the treatment of deep vein thrombosis and pulmonary embolism and long-term secondary prevention of venous thromboembolism, and for stroke and systemic embolism prevention in patients with nonvalvular atrial fibrillation. Rivaroxaban has many advantages over vitamin $\mathrm{K}$ antagonists and this may facilitate its use in clinical practice. As a result, it is expected that new oral anticoagulants may change patient management strategies. On the other hand, rivaroxaban has some particularities that are necessary to know. The aim of this manuscript was to review the use of rivaroxaban not only in general population, but also in specific patients groups and clinical situations to achieve an optimal management with this drug in daily clinical practice.

Key words. Anticoagulation. Atrial fibrillation. Deep vein thrombosis. Rivaroxaban. Stroke. Treatment. Venous thromboembolism. 